\title{
Estudio observacional del ojo seco en una farmacia
} comunitaria

\author{
Antucho Espinosa Estévez', Antucho Espinosa Suances ${ }^{2}$ \\ 1. Farmacéutico comunitario en A Gudiña, Ourense. Graduado en Óptica, Optometría y Audiología. Máster en Biotecnología. \\ 2. Farmacéutico comunitario en A Valenzá, Ourense. Máster en Atención Farmacéutica.
}

\section{PALABRAS CLAVE}

Síndrome de ojo seco, farmacia comunitaria, lágrimas artificiales, adherencia

\section{ABREVIATURAS}

DE: desviación estándar FC: farmacia comunitaria LA: lágrimas artificiales SNS: Sistema Nacional de Salud SOS: Síndrome de 0jo Seco

\section{KEYWORDS}

Dry eye disease, community pharmacy, artificial tears, compliance

\section{RESUMEN}

Introducción: El síndrome de ojo seco (SOS) afecta a cinco millones de personas en España y a sesenta millones en todo el mundo, acarreando importantes costes y afectando a actividades diversas. Puede ser de tipo acuodeficiente o evaporativo y el diagnóstico se lleva a cabo según la percepción del paciente, seguida de pruebas objetivas. No tiene curación y el tratamiento va siempre enfocado a paliar los síntomas.

Objetivos: Conocer la prevalencia del SOS en A Gudiña (Ourense). Además, se determinó en qué grado la falta de adherencia afecta al tratamiento con lágrimas artificiales (LA); así como la asociación entre el SOS y ciertas variables analizadas.

Material y métodos: Durante los siete meses de duración del estudio, se seleccionó una muestra final de 51 pacientes de SOS. Los datos se recogieron cumplimentando una hoja de registro diseñada ad hoc.

Resultados: La prevalencia de SOS en A Gudiña es relativamente baja (4,3\%). La media de edad es de 66 años, afectando mayoritariamente a mujeres. Se detectó una falta de adherencia al tratamiento con LA. Todas las variables analizadas mostraron asociación con la enfermedad.

Conclusiones: La prevalencia de síndrome de ojo seco en A Gudiña es ligeramente menor que la encontrada en la literatura. La exclusión de las LA del seguro médico es la causa principal de la falta de adherencia a los tratamientos. De los antecedentes patológicos valorados en este estudio, la cirugía ocular es el que más afecta en el desarrollo del SOS.

Observational study of dry eye in community pharmacy

\section{ABSTRACT}

Introduction: Dry Eye Disease (DED) is a multifactorial symdrom. It affects five million people in Spain and sixty million persons around the world, carrying significant costs and affecting different activities. DED can be aqueous-deficient or evaporative and diagnosis is performed according to the patient's symptoms, followed by objective tests. It is a chronic disease; therefore, treatment is always focused on alleviating symptoms.

Objectives: The main purpose of this study was to set DED prevalence in A Gudiña (a municipality in the province of Ourense). Additionally, it was determined to what extent it is affected by the lack of adherence to the treatment with artificial tears (AT) and the association between DED and certain variables analyzed.

Materials and methods: During the seven months of the study, a final sample of 51 DED patients was selected. Data were collected by completing a registration form designed ad hoc.

Results: DED prevalence in A Gudiña is relatively low (4.25\%). The average age being 66 , it was proved to affect mostly women. A lack of adherence to the treatment with AT was reported. All variables analyzed showed association with the disease.

Conclusions: DED prevalence in A Gudiña is lower than that found in the literature. The exclusion of AT from health insurance is the main cause of low adherence to treatment. From the set of the medical variables analyzed, eye surgery is the main source of DED development. 


\section{Introducción}

De todas las enfermedades oculares, el síndrome de ojo seco (SOS) es quizás la más esquiva. Durante años la definición ha variado hasta que en 2007 se logró un consenso para definirlo como una enfermedad multifactorial de la película lacrimal y la superficie ocular que causa sintomas de malestar, trastornos visuales e inestabilidad de la película lacrimal con un daño potencial en la superficie ocular; va acompañada por un incremento de la osmolaridad de la película lacrimal e inflamación de la superficie ocular. Dada la relación directa entre el SOS y la lágrima es también conocido como Síndrome de Disfunción Lagrimal (1).

De las múltiples clasificaciones posibles para el SOS, las dos más extendidas son las efectuadas según las causas que lo originan y la gravedad de la afección.

- La clasificación etiológica distingue el SOS acuodeficiente del evaporativo, pudiendo coexistir y estar originados a su vez por múltiples causas. Las más frecuentes son la disfunción de la glándula, la obstrucción del conducto lacrimal, el bloqueo reflejo, los efectos secundarios de ciertos fármacos, la disfunción de las glándulas de Meibomio, las alteraciones del párpado y el uso de lentes de contacto (2).

- En base a la clasificación fisiopatológica esta enfermedad puede ir evolucionando gradualmente según síntomas y signos (3).

Los dos mecanismos fisiopatológicos subyacentes son la hiperosmolaridad y la inestabilidad de la película lagrimal (4). La hiperosmolaridad se origina como consecuencia de una excesiva evaporación de agua de la superficie ocular y estimula una cascada de eventos inflamatorios en las células epiteliales. La inestabilidad de la película lagrimal da lugar a desecación local, alteración del glicocalix y mucinas de las células caliciformes, así como a la disminución de lisozima y lactoferrina en la lágrima (1).

Los datos epidemiológicos dictan que el SOS es la enfermedad más frecuente en oftalmología (5). En un estudio realizado España con una población mayor de 40 años, se encontró una prevalencia del 11\%; lo que viene a ser en términos numéricos, cinco millones de españoles (4). A nivel mundial los datos de prevalencia no están del todo claros, dependen de la definición de ojo seco usada, de la distribución geográfica donde se evalúe y del rango de edad de la población analizada $(4,6)$.

Los sintomas del paciente de SOS son los típicos de sequedad ocular: sensación de cuerpo extraño, quemazón, percepción de arenilla en los ojos, escozor, picor, pesadez palpebral e irritación. Estos síntomas pueden verse agravados en ambientes de baja humedad relativa en los que aumenta la evaporación de la lágrima, como exposición a aire acondicionado, calefacción, humos o viento, y en determinadas épocas del año según la humedad ambiental (7).

El diagnóstico y seguimiento clínico del paciente con este sindrome supone un gran desafío, ya que no parece haber una clara correlación entre signos y sintomas (4). En consecuencia, se recomienda realizar una combinación de pruebas con el fin de determinar la etiología y la gravedad en cada caso. Los síntomas del paciente se evalúan mediante cuestionarios validados (8). Su mayor inconveniente es su falta de correlación con los sintomas de la enfermedad (9). Los signos clínicos se determinan llevando a cabo una serie de pruebas, todas ellas enfocadas a la evaluación de la cantidad y calidad de la película lacrimal, párpados y superficie ocular (8).

El tratamiento de esta enfermedad suele ser bastante frustrante, ya que normalmente no tiene cura, persistiendo de por vida y variando según la gravedad del proceso. Para tratar los casos de SOS leve y moderado se recurre a medidas higiénicas (alimentos saludables, parpadeo forzado, evitar corrientes de aire, higiene palpebral diaria...) y a la instilación de lágrimas artificiales (LA) (10). El SOS grave necesita un tratamiento más agresivo, normalmente con antiinflamatorios, inmunomoduladores y secretagogos (11). Los casos complicados se tratan con suero autólogo y mediante la oclusión de los puntos lacrimales $(10,12)$.

La mayoría de los estudios publicados hasta la fecha sobre el SOS se refieren a la fisiopatología, diagnóstico y tratamiento de la enfermedad, siendo escasos los realizados en farmacia comunitaria $(\mathrm{FC})(13,14,15)$.
Los datos de prevalencia son preocupantes, con el agravante de que se estima que existen treinta y tres millones de adultos sin diagnosticar en el mundo (4). En el ámbito de la salud pública es una patología incapacitante, con un impacto significativo en cuanto a calidad de vida se refiere. La situación se complica a partir del año 2012, cuando las LA quedan excluidas de la prestación farmacéutica del Sistema Nacional de Salud (SNS). Por lo tanto, la consulta médica que originaba la prescripción de estos medicamentos está siendo sustituida por la automedicación o, en el mejor de los casos, por la indicación farmacéutica. Ello, aumenta la responsabilidad del farmacéutico al ser el único profesional sanitario que tiene constancia de la utilización de muchos de estos medicamentos. Todas estas circunstancias motivan que se lleve a cabo el presente estudio.

\section{Objetivos}

- Conocer el grado de prevalencia del SOS entre los pacientes de una farmacia comunitaria.

- Establecer la posible relación entre el uso de determinados medicamentos y otras situaciones fisiológicas o patologías con el desarrollo de la enfermedad.

- Determinar el grado de adherencia al tratamiento con LA.

\section{Material y métodos}

\section{Tipo de estudio, ámbito local y} temporal

Estudio observacional transversal realizado en una farmacia comunitaria única del municipio de A Gudiña (Ourense), de 1.584 habitantes, entre el 1 de junio de 2014 y el 31 de diciembre de 2014. El período de estudio se estableció en 7 meses con el fin de que pasasen por la farmacia el mayor número posible de pacientes del municipio que cumplian los criterios de inclusión.

\section{Sujetos}

Criterios de inclusión: pacientes que en el periodo de estudio acudieron a la farmacia a recoger sus lágrimas artificiales, geles oculares o lubricantes oculares para el tratamiento de la afección, o bien estaban a tratamiento con uno o varios fármacos 
sistémicos o tópicos oculares, que podrían estar implicados en el desarrollo del ojo seco y que dieron su consentimiento informado para participar en el estudio.

Criterio de exclusión: padecer una incapacidad física o psíquica que impidiese al sujeto participar en el estudio.

La población de partida son los 1.584 habitantes del municipio. La población accesible estuvo constituida por 1.200 individuos, que en el periodo analizado acudieron sin dificultad a la farmacia. De éstos, se detectaron 51 que cumplían los criterios de inclusión y, por lo tanto, constituyeron la muestra objeto de estudio.

\section{Variables}

- Edad.

- Sexo.

- Menopausia: pacientes que muestran los primeros síntomas de ojo seco en la perimenopausia.

- Uso de lentes de contacto.

- Antecedentes patológicos personales.

- Medicamentos: tratamientos farmacológicos habituales en los pacientes que son considerados factores de riesgo de SOS.

- Adherencia al tratamiento con LA, determinando en cada caso particular si se da una baja adherencia y su causa correspondiente.

La recogida de datos se realizó mediante una hoja de registro diseñada ad hoc (figura 1). Dicho cuestionario fue administrado por los farmacéuticos que trabajan en la farmacia.

Las variables cuantitativas se expresan como medias (DE) (rango) y las cualitativas como proporciones.

\section{Resultados}

Se localizaron 51 pacientes que cumplían los criterios de inclusión y que confirmaron la existencia de un diagnóstico de síndrome de ojo seco, por lo que la prevalencia de esta patología en la población de A Gudiña se estimó en el 4,3\%. La edad media de dichos pacientes fue de 69,6 años $(\mathrm{DE}=15,5)$ (13-91). 39 (76,5\%) mujeres, 5 (12,8\%) de las cuales sufren ojo seco en edad perimenopáusica.

$23(45,1 \%)$ pacientes presentaron alguna patología con riesgo de

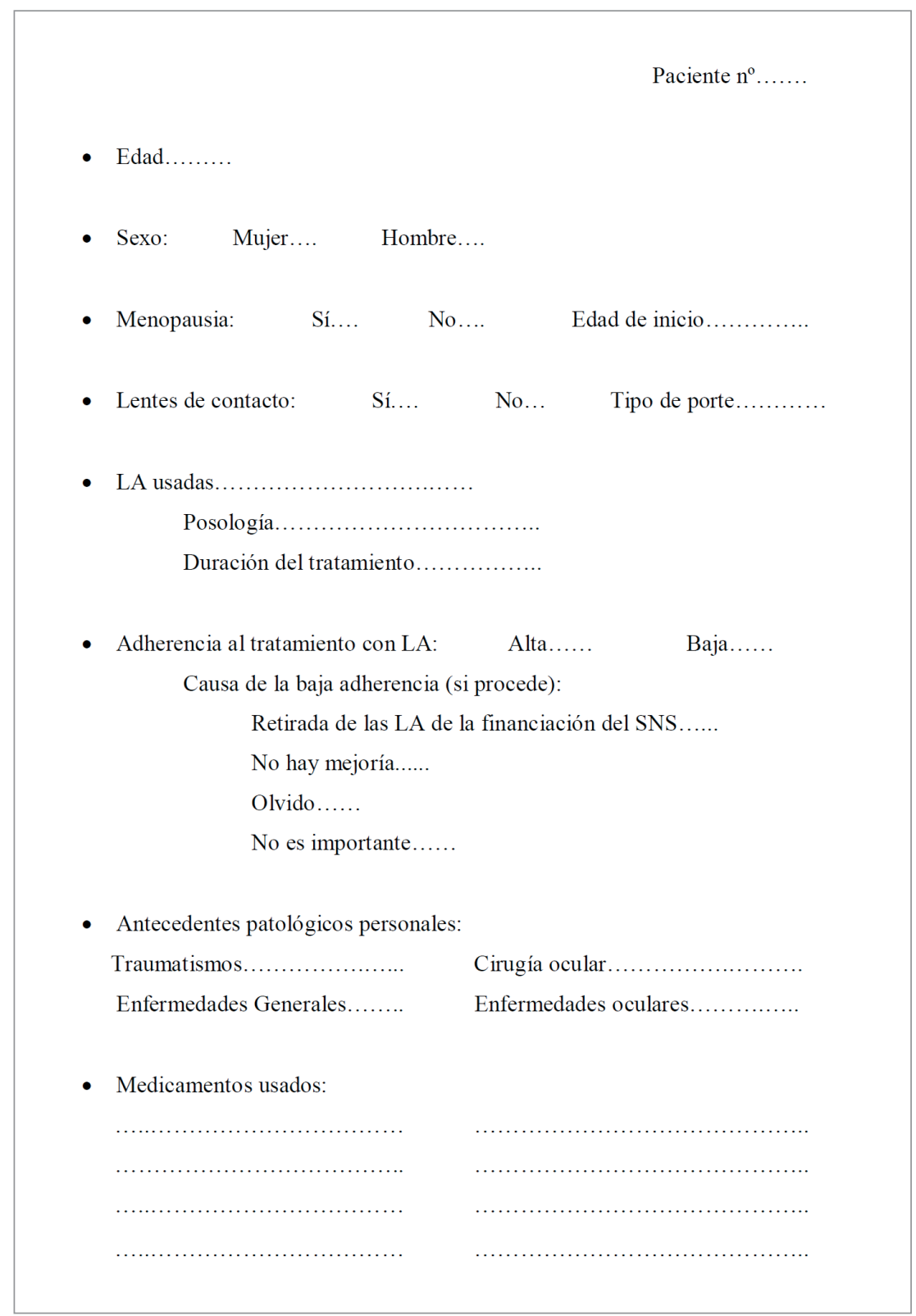

Figura 1 Cuestionario utilizado

desencadenar SOS. Se distribuyen en 4 grupos, tal como se muestra en la (figura 2); encontrándose los siguientes resultados: en 1 individuo se asoció a un traumatismo ocular, en 3 a una enfermedad general, en 17 a una cirugía ocular y en 2 a una enfermedad ocular.

Se ha detectado un único caso que relacione el uso de las lentes de contacto con el avance de la enfermedad, que corresponde a una mujer de 41 años.

Tras revisar la medicación activa de cada uno de los 51 pacientes, se detectaron un total de 52 medicamentos considerados factor de riesgo de SOS; ello supone una media de $1,0(\mathrm{DE}=1,0)$ fármaco por paciente. $\mathrm{Su}$ distribución por grupo terapéutico es la siguiente: 15 pacientes en
Figura 2 Causas de la mala adherencia al tratamiento

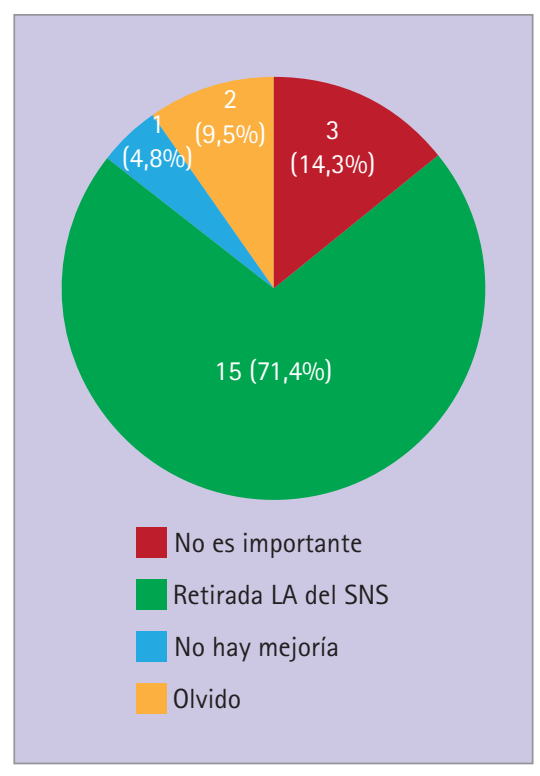




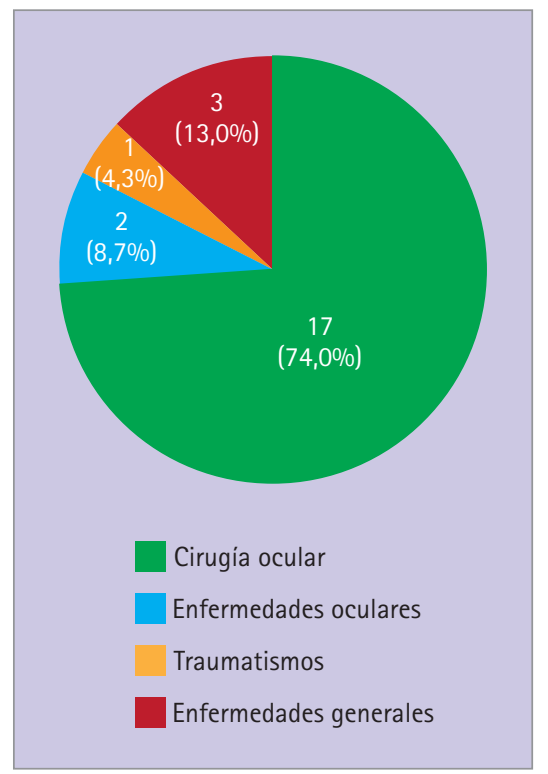

Figura 3 Antecedentes patológicos personales de los sujetos objeto de estudio

tratamiento con diuréticos, 13 con antidepresivos, 11 con betabloqueantes, 7 con antihistamínicos, 4 con anticolinérgicos y 2 con metotrexato.

$21(41,2 \%)$ sujetos tienen una mala adherencia al tratamiento con LA. Las causas de la mala adherencia se recogen en la (figura 3 ) y se corresponden con los siguientes resultados encontrados: en 15 pacientes está asociada a la retirada de las LA del SNS, 1 no notó mejoría, 2 se olvidaban de usarlas y 3 no las consideraban importantes.

\section{Discusión}

Al trabajar con una muestra relativamente pequeña (51 sujetos), los resultados obtenidos no son representativos para darles un tratamiento estadístico ya que presentan un error muestral del $13,4 \%$ para un nivel de confianza del 95\% (máximo nivel de precisión posible al incluir a todos los pacientes), lo cual supone una limitación muy importante. Sin embargo, el mayor interés de este trabajo radica en que se estudia esta patología en los individuos del municipio y en que se realiza en el ámbito de la FC.

La prevalencia encontrada está por debajo del rango real de la enfermedad, que oscila de 5-30\% (2), más infravalorada, aún si cabe, en este estudio por el hecho de tener en cuenta únicamente pacientes diagnosticados y en tratamiento con LA, descartando una fracción significativa de pacientes asintomáticos.
Atendiendo al tipo de población a estudio y a las condiciones ambientales de la región, dicho valor se encuentra dentro de la normalidad, dado que el SOS tiene considerablemente menos impacto en la localidad estudiada que en otras poblaciones con clima más seco y con cambios bruscos de temperatura a lo largo del año. De hecho, un estudio realizado en Valladolid con 278 sujetos ofrece índices de prevalencia bastante superiores (16).

La media de edad es concordante con lo recogido en publicaciones anteriores, donde se evidencia un aumento de la patología con la edad $(16,17)$.

Respecto al sexo, los resultados del estudio indican que la mujer es, sin lugar a dudas, y coincidiendo con otros trabajos publicados (1), la afectada mayoritariamente por el SOS. En todas las mujeres los síntomas que acompañan a la menopausia habrían comenzado en dicha etapa y se mantendrían a lo largo de su vida, por lo tanto es en estos casos el factor etiológico principal en la fisiopatogenia de la enfermedad.

El que se detectase un único caso que asocie el uso de lentes de contacto con la evolución de la enfermedad parece un dato sorprendentemente bajo, sobre todo si tenemos en cuenta la alta incidencia de SOS en portadores de lentillas $(18,19)$. Si bien es cierto que el empleo de lentes de contacto no está extendido entre los sujetos del estudio por tratarse de una población eminentemente rural y con una media de edad relativamente alta.

De las diferentes patologías con riesgo de desencadenar SOS, las distintas modalidades de cirugía del ojo son la causa predominante al igual que en otros estudios $(20,21)$. Por otra parte, los 6 grupos farmacológicos de medicamentos hallados en el estudio parecen relacionarse con la enfermedad, tal como se observa en otros estudios anteriormente publicados (22).

Se encontraron valores altos de incumplimiento del tratamiento con LA, no relacionado con la efectividad, que, dada la simplicidad de la administración del fármaco, resultaba de vital importancia averiguar las causas reales. La retirada de las LA de la cobertura del SNS resultó ser la más importante, con mucha diferencia respecto a las demás. La situación de crisis económica que está vivien- do la sociedad española, sumada a la exclusión de las LA del SNS, pone de manifiesto un problema actual: muchos pacientes no logran afrontar los gastos económicos que suponen dichos tratamientos no financiados. La consecuencia de una falta de adherencia a largo plazo traería consigo una situación cronificada de la enfermedad, lo cual supone un factor de riesgo para otras patologías oculares de mayor gravedad. Por lo tanto, el hecho de no tratar adecuadamente un problema menor, que tendría unos costes perfectamente soportables para el SNS, probablemente acabe desarrollando una patología mayor y con unos costes superiores. En consecuencia, el papel que tiene que jugar el farmacéutico comunitario para paliar esta situación y mejorar los resultados del tratamiento del SOS es capital, dejando la puerta abierta a futuros trabajos en este campo.

\section{Conclusiones}

- La prevalencia de síndrome de ojo seco en el municipio de A Gudiña es ligeramente menor que la encontrada en la literatura.

- Se ha observado una posible relación entre los grupos farmacológicos evaluados y el Sos.

- De los antecedentes patológicos personales valorados en este estudio, la cirugía ocular es la patología más importante en el desarrollo del SOS.

- La exclusión de las LA de la prestación del SNS ha sido la causa principal de la falta de adherencia a los tratamientos.

\section{Referencias bibliográficas}

1. Lemp MA, Baudouin C, Baum J, Dogru M, Foulks GN, Kinoshita S, et al. The definition and classification of dry eye disease: report of the Definition and Classification Subcommittee of the International Dry Eye WorkShop. Ocul Surf. 2007; 5(2):75-79. doi:10.1016/S1542-0124(12)70081-2

2. Gipson IK, Argüeso P, Beuerman R, Bonini S, Ham B, Jumblatt M et al. The investigation of dry eye disease: report of the Investigation Subcommittee of the International Dry Eye WorkShop. Ocul Surf. 2007; 5(2):184-198.

3. Santodomingo J. Ojo seco. Gaceta Óptica. 2009; 426:20-27.

4. Viso E, Rodríguez-Ares MT, Abelenda D, Oubiña B, Gude F. Revalence of asymptomatic and symptomatic 
meibomian gland dysfunction in the general population of Spain. Invest Ophthalmol Vis Sci. 2012; 53:26012006. doi:10.1167/iovs.11-9228

5. Díaz Valle D. XV Encuentro Nacional de Salud y Medicina de la Mujer. Colegio de Médicos de Madrid. Síndrome de ojo seco y degeneración macular. Madrid: Colegio Médico; 2015.

6. Moss SE, Klein R, Klein BE. Incidence of dry eye in an older population. Arch Ophthalmol. 2004; 122(3):369373. doi:10.1001/archopht.122.3.369

7. Alves M, Novaes P, Morraye Mde A, Reinach PS, Rocha EM. Is dry eye an environmental disease? Arq Bras Oftalmol. 2014; 77(3):193-200. doi:10.5935/0004-2749.20140050

8. Fuentes-Páeza, J.M. Herrerasb, Y. Cordero, A. Falta de concordancia entre los cuestionarios y las pruebas diagnósticas en el síndrome de ojo seco. Arch Soc Esp Oftalmol. 2011; 86(1):3-7. doi:10.1016/j.oftal.2010.07.004

9. Benítez del Castillo JM, Vico E. Tratamiento del ojo seco. Gaceta Óptica. 2004; 385:10-14.

10. Messmer ME. The Pathophysiology, Diagnosis, and Treatment of Dry Eye Disease. Dtsch Arztebl Int. 2015 112(5):71-82.

11. Geerling G, Kinoshita S, Lemp MA, McCulley, Nelson D, Novack GN et al. Management and Therapy of Dry Eye
Disease: Report of the Management and Therapy Subcommittee of the International Dry Eye Work Shop. Ocul Surf. 2007; 5(2):168-183.

12. Herrero-Vanrell R, Molina-Martínez I. Autologous serum in the treatment of dry eye sindrome. Technological aspects. Arch Soc Esp Oftalmol. 2008; 83:521-524.

13. Román MT, Jarque MJ, Salgado T, García L, Navarro JA, Maeso I. Ojo seco en la Oficina de Farmacia. Colegio Oficial de Farmacéuticos de Valladolid y Vocalía de Óptica y Acústica. XVII Congreso Nacional Farmacéutico (A0-01). Bilbao; 2010.

14. Román T, Blas L, Bronte JI, Caballero A, Coello F, Cotón MA et al. Ojo seco en la Oficina de Farmacia. Consejo General de Colegios Oficiales de Farmacia. XVII Congreso Nacional Farmacéutico (A0-02). Bilbao; 2010.

15. Puchol SV, Beltrán V, Fuster A, Tamarit L, Sanchís R, Sanfeliu M. Actuación del farmacéutico comunitario ante el síndrome de ojo seco. V Congreso de Atención Farmacéutica. Oviedo; 2007.

16. Herreras J, Fuentes-Páez G, Cordero Y, Almaraz A, Calonge M. Prevalence of dry eye syndrome in Valladolid (Spain). ARVO 2006, E abstract 260.

17. Chia EM, Mitchell P, Rochtchina E, Lee AJ, Maroun R, Wang JJ. Prevalence and associations of dry eye sy- ndrome in an older population: the Blue Mountains Eye Study. Clin Experiment Ophthalmol. 2003; 31: 229232. doi:10.1046/j.1442-9071. 2003. 00634. $\mathrm{x}$

18. Chalmers RL, Begley CG. Dryness symptoms among an unselected clinical population with and without contact lens wear. Contact Lens \&t Anterior Eye. 2006; 29:25-30. doi:10. 1016/j.clae.2005.12.004

19. Chalmers RL, Hunt C, Hickson-Curran S, Young G. Struggle with hydrogel CL wear increases with age in young adults. Cont Lens \& Anterior Eye. 2009; 32:113-119. doi:10.1016/j. clae.2008.12.001

20. Kasetsuwan N, Satitpitakul V, Changul $\mathrm{T}$, Jariyakosol $\mathrm{S}$. Incidence and Pattern of Dry Eye after Cataract Surgery. PLoS One. 2013; 8(11). doi:10.1371/journal.pone.0078657

21. Levitt AE, Galor A, Weiss JS, Felix ER, Martin ER, Patin DJ et al. Chronic dry eye symptoms after LASIK: parallels and lessons to be learned from other persistent post-operative pain disorders. Mol Pain. 2015; 11:21. doi:10.1186/s12990015-0020-7

22. Fraunfelder FT, Sciubba JJ, Mathers WD. The Role of Medications in Causing Dry Eye. Journal of Ophthalmology. 2012, Article ID 285851, 8 pages. doi:10.1155/2012/285851 